\title{
SIMULTANEOUS SURFACE RESOLUTION IN CYCLIC GALOIS EXTENSIONS
}

\author{
SHREERAM S. ABHYANKAR AND NAN GU \\ (Communicated by Ted Chinburg)
}

\begin{abstract}
We show that simultaneous surface resolution is not always possible in a cyclic extension whose degree is greater than three and is not divisible by the characteristic. This answers a recent question of Ted Chinburg.
\end{abstract}

\section{INTRODUCTION}

Let $K$ be a two dimensional algebraic function field over an algebraically closed ground field $k$. Recall that $K / k$ has a minimal model means that amongst all the nonsingular projective models of $K / k$ there is one which is dominated by all others (basic reference $[\mathrm{Ab} 4]$ or $[\mathrm{Ab} 5]$ ). Also recall that $K / k$ has a minimal model if and only if it is not a ruled function field, i.e., $K$ is not a simple transcendental field extension of a one dimensional algebraic function field over $k$ (see [Zar]). A finite algebraic field extension $L / K$ is said to have a simultaneous resolution if there exist nonsingular projective models $V$ and $W$ of $K / k$ and $L / k$, respectively, such that $W$ is the normalization of $V$ in $L$. Given any positive integer $q$ that is not divisible by the characteristic $\operatorname{char}(K)$ of $K$ and letting $Z_{q}$ denote a cyclic group of order $q$, in [Ab2] it was shown that if $q \leq 3$ and $L / K$ is a $Z_{q}$ extension, i.e., a Galois extension whose Galois group is a cyclic group of order $q$, then it has a simultaneous resolution, whereas if $K / k$ has a minimal model and $q>3$ with $q$ being a prime number, then there exists a $Z_{q}$ extension $L / K$ that has no simultaneous resolution. Here we shall extend this second result to those nonprimes $q$ that are divisible by the square of some prime $p$. By taking $q=4$, this answers a question raised by Ted Chinburg at the March 2006 AMS Meeting in New Hampshire to the effect whether every $Z_{2}$ by $Z_{2}$ extension $L / K$, i.e., a $Z_{2}$ extension $L / J$ of a $Z_{2}$ extension $J / K$, has a simultaneous resolution. By using a theorem of David Harbater and Florian Pop, we generalize our extended result by replacing $Z_{q}$ by its direct sum $H \oplus Z_{q}$ with any finite group $H$. For a related matter, see [AbK].

In Lemma (2.2) of Section 2 we shall give a consequence of the Harbater-Pop Theorem to be used in proving our generalized extended result. In Lemma (2.1) of Section 2 we shall summarize some technical results from our previous papers [Ab2] and [Ab3]. These technical results deal with the structure of the integral closure of a normal noetherian domain in a cyclic extension. They are used in the proof of Theorem (3.1) of Section 3, which gives a sufficient condition for a two dimensional

Received by the editors August 28, 2006 and, in revised form, November 9, 2006.

2000 Mathematics Subject Classification. Primary 14A05.

(C) 2007 American Mathematical Society Reverts to public domain 28 years from publication 
local domain to be nonregular. Theorem (3.1) is used in proving the special case of Theorem (3.2) of Section 3, which corresponds to our extended result, i.e., the $H=1$ case of our generalized extended result. The general case of Theorem (3.2), which corresponds to our generalized extended result, then follows by using Lemma (2.2).

\section{TWO LEMMAS}

Let $M(R)$ denote the maximal ideal of a local ring $R$. In Lemma (2.1) we summarize some properties of the integral closure of a normal noetherian domain in a cyclic extension. In Lemma (2.2) we give a consequence of the Harbater-Pop theorem.

Lemma 2.1. Let $R$ be a normal noetherian domain with quotient field $K$, let $S$ be the integral closure of $R$ in a finite algebraic field extension $L$ of $K$, and let $[L: K]=q$. Assume that $q$ is a unit in $R$ and that $L$ contains a nonzero element $z$ such that $L=K(z)$ and

$$
z^{q}=u \prod_{j=1}^{d} x_{j}^{a(j)}
$$

where $u$ is a unit in $R, d$ is a nonnegative integer, $a(j)$ is an integer such that $\operatorname{GCD}(a(j), q)=1$ for $1 \leq j \leq d$, and $x_{1}, \ldots, x_{d}$ are elements in $R$ such that $x_{1} R, \ldots, x_{d} R$ are pairwise distinct minimal (= height one) prime ideals in $R$. Let $b(i, j)$ and $c(i, j)$ be the unique integers such that

$$
\left.b(i, j)=a(j) i+c(i, j) q \quad \text { and } \quad 0 \leq b_{(} i, j\right)<q .
$$

Let

$$
z_{i}=z^{i} \prod_{j=1}^{d} x_{j}^{c(i, j)}
$$

Then we have the following:

(1) $\left(z_{0}, \cdots, z_{q-1}\right)$ is a free $R$-basis of $S$.

(2) If $R$ is a local domain and $d \geq 1$, then $S$ is a local domain and for its maximal ideal $M(S)$ we have $M(S)=M(R) S+\left(z_{1}, \cdots, z_{q-1}\right) S$ with $S / M(S)=R / M(R)$

(3) If $R$ is a regular local domain and $d \geq 2$, then $S$ is a nonregular local domain.

Proof. For (1) and (2) see Theorem 7 [Ab3]. For (3) see Theorem 6 [Ab2] with the observation that, although in the context of this theorem $q$ is a prime number, the primeness of $q$ was never used in its proof. A different version of (1) and (2) can also be found in Theorems 4 and 5 [Ab2]; see Remark 2 on page 28 of [Ab3].

Lemma 2.2. Let $K / k$ be a two dimensional algebraic function field over an algebraically closed ground field $k$. For any finite group $H$, there exists a Galois extension $\widetilde{L} / K$ with Galois group $H$.

Proof. It follows from Theorem 4.4 [Har] or the Corollary to Theorem A [Pop] that given any finite group $H$ and any one dimensional algebraic function field $E$ over an algebraically closed ground field $k$, there exists a Galois extension $F / E$ whose Galois group is $H$. The following argument, provided by Harbater and Pop, shows how the desired two-variable existence follows from this. 
Given a two dimensional algebraic function field $K$ over $k$, choose a separating transcendence basis $x, y$ for $K$ over $k$. So $K$ is a finite separable field extension of $k(x, y)$. Let $E$ be the algebraic closure of $k(x)$ in $K$. $E$ is finite over $k(x)$, since $K$ is finite over $k(x, y)$ and since $k(x)$ is algebraically closed in $k(x, y)$. Thus $E$ is a one dimensional algebraic function field over $k$ and so, by the one-variable existence theorem, $H$ is the Galois group of a finite extension $F$ of $E$. Since $E$ is algebraically closed in $K$ and since $F$ is algebraic over $E$, it follows that $F$ and $K$ are linearly disjoint over $E$. So the compositum $\widetilde{L}=K F$ (in an algebraic closure of $K$ ) is a Galois extension of $K$ with Galois group $H$, completing the proof.

\section{TWO THEOREMS}

In Theorem (3.1) we give a sufficient condition for a local domain to be nonregular. In Theorem (3.2) we construct our examples of simultaneous nonresolvability.

Theorem 3.1. Let $R$ be a two dimensional regular local domain, let $(X, Y)$ be generators of its maximal ideal $M(R)$, and let $K$ be its quotient field. Let $R_{0}=R$. For all $n>0$, let $Y_{n}=Y / X^{n}$ and let $R_{n}$ be the localization of the ring $R_{n-1}\left[Y_{n}\right]$ at the maximal ideal in it generated by $\left(X, Y_{n}\right)$. Note that then $R_{n}$ is a two dimensional regular local domain with quotient field $K$ such that $R_{n}$ dominates $R_{n-1}$ and $\left(X, Y_{n}\right)$ are generators of $M\left(R_{n}\right)$.

Let $q$ be a positive integer that is a unit in $R$. Assume that $q=p m$ where $p$ is a prime number and $m$ is a positive integer divisible by $p$. Assume that $K$ contains $q$ distinct $q$-th roots of 1 . Let $L$ be a splitting field over $K$ of the polynomial of $Z^{q}-X Y^{m}$. Let $S_{n}$ be the integral closure of $R_{n}$ in $L$.

Then $L / K$ is a $Z_{q}$ extension and for every nonnegative integer $n$, the ring $S_{n}$ is a two dimensional nonregular local domain.

Proof. Let $w$ be the discrete valuation whose valuation ring is the one dimensional regular local domain obtained by localizing the $\operatorname{ring} R$ at the prime ideal in it generated by $X$. Then $w\left(X Y^{m}\right)=1$ and hence the polynomial $Z^{q}-X Y^{m}$ is irreducible in $K[Z]$ and $L / K$ is a $Z_{q}$ extension. Let $z \in L$ be a root of the said polynomial. Then $z^{q}=X Y^{m}$ and $L=K(z)$. Let $\bar{X}=z^{p} / Y$ and $J=K(\bar{X})$. Then $\bar{X}^{m}=X$ and hence $J / K$ is a $Z_{m}$ extension. By (2.1)(2) the integral closure $T_{n}$ of $R_{n}$ in $J$ is a two dimensional regular local domain whose maximal ideal $M\left(T_{n}\right)$ is generated by $\left(\bar{X}, Y_{n}\right)$. Also $z^{p}=\bar{X} Y=\bar{X}^{1+n m} Y_{n}$ and, since $m$ is assumed divisible by $p$, upon letting $\zeta=z / \bar{X}^{n m / p}$ we get $L=J(\zeta)$ with $\zeta^{p}=\bar{X} Y_{n}$. Now $L / J$ is a $Z_{p}$ extension with $L=J(\zeta)$, and $S_{n}$ is the integral closure of $T_{n}$ in $L$. Therefore by $(2.1)(3)$ we see that $S_{n}$ is a two dimensional nonregular local domain.

Theorem 3.2. Let $K / k$ be a two dimensional algebraic function field over an algebraically closed ground field $k$. Assume that $K / k$ has a minimal model $V^{*}$. Let $q$ be a positive integer that is not divisible by $\operatorname{char}(K)$. Assume that $q=$ pm where $p$ is a prime number and $m$ is a positive integer divisible by $p$. Then, given any finite group $H$, there exists a Galois extension $L^{\prime} / K$ with Galois group $H \oplus Z_{q}$ such that $L^{\prime} / K$ has no simultaneous resolution.

Proof. By (2.2) there exists a Galois extension $\widetilde{L} / K$ with Galois group $H$. Take $R$ in (3.1) to be the local ring of a point of $V^{*}$ that is not ramified in $\widetilde{L}$. Let $L^{\prime}$ be a compositum of $\widetilde{L}$ and $L$. It is easy to see that $L^{\prime} / K$ is a Galois extension whose Galois group is $H \oplus Z_{q}$. 
By [Ab1, Lemma 12], there exists a unique valuation $v$ of $K$ dominating $R_{n}$ for all $n \geq 0$. By construction each $R_{n+1}$ is the immediate quadratic transform of $R_{n}$ along $v$. Let $\widetilde{v}$ be an extension of $v$ to $\widetilde{L}$. Let, if possible, $V$ and $W$ be nonsingular projective models of $K / k$ and $L^{\prime} / k$, respectively, such that $W$ is the normalization of $V$ in $L^{\prime}$. Then by the minimality of $V^{*}, V$ must dominate $V^{*}$. Consequently by [Ab1, Theorem 3] the local ring of the center $P$ of $v$ on $V$ must equal $R_{n}$ for some nonnegative integer $n$. Since $R_{n}$ dominates $R$ and $R$ is not ramified in $\widetilde{L}, R_{n}$ is not ramified in $\widetilde{L}$. Let $\widetilde{V}$ be the normalization of $V$ in $\widetilde{L}$, and $\widetilde{R}_{n}$ be the local ring of the center $\widetilde{P}$ of $\widetilde{v}$ on $\widetilde{V}$. Then $\widetilde{P}$ lies above $P$ in $\widetilde{V}$ and $\widetilde{R}_{n}$ is a two dimensional regular local ring whose maximal ideal $M\left(\widetilde{R}_{n}\right)$ is generated by $\left(X, Y_{n}\right)$. Now $L^{\prime}$ is a $Z_{q}$ extension of $\widetilde{L}$ constructed from $\widetilde{L}$ in the same way as $L$ is constructed from $K$ in (3.1), and $W$ is the normalization of $\widetilde{V}$ in $L^{\prime}$. By (3.1), the point of $W$ lying above $\widetilde{P}$ is not a simple point, which is a contradiction.

Remark 3.3. The construction of a $Z_{q}$ extension $L / K$ having no simultaneous resolution does not use the results of Harbater and Pop. Their results plus the fact that a regular system of parameters lifts to a regular system of parameters through an unramified local ring extension allow us to mimic such a construction to get an $H \oplus Z_{q}$ extension. Similar arguments will show that the statement of (3.2) remains true if $q>3$ is a prime number; see [Ab2, Theorem 11] for details.

\section{References}

[Ab1] S. S. Abhyankar, On the valuations centered in a local domain, American Journal of Mathematics, 78 (1956), 321-348. MR0082477 (18:556b)

[Ab2] S. S. Abhyankar, Simultaneous resolution for algebraic surfaces, American Journal of Mathematics, 78 (1956), 761-790. MR0082722 (18:600b)

[Ab3] S. S. Abhyankar, Uniformization of Jungian local domains, Mathematische Annalen, 159 (1965), 1-43. MR0177989 (31:2247)

[Ab4] S. S. Abhyankar, Resolution of Singularities of Embedded Algebraic Surfaces, Springer Verlag (1998). MR1617523 (99c:14021)

[Ab5] S. S. Abhyankar, Lectures on Algebra I, World Scientific, 2006.

$[\mathrm{AbK}]$ S. S. Abhyankar and M. Kumar, Simultaneous surface resolution in quadratic and biquadratic Galois extensions, Contemporary Mathematics, 390 (2005), 1-8. MR2187320 (2006h:14017)

[Har] D. Harbater, Fundamental groups and embedding problems in characteristic p, Contemporary Mathematics, 186 (1995), 353-369. MR1352282 (97b:14035)

[Pop] F. Pop, Étale Galois covers of affine smooth curves, Invent. Math., 120 (1995), 555-578. MR1334484 (96k:14011)

[Zar] O. Zariski, The problem of minimal models in the theory of algebraic surfaces, American Journal of Mathematics, 80 (1958), 146-184. MR0097404 (20:3873)

Department of Mathematics, Purdue University, West Lafayette, Indiana 47907

E-mail address: ram@cs.purdue.edu

Department of Mathematics, Purdue University, West Lafayette, Indiana 47907

E-mail address: ngu@math.purdue.edu 